\title{
Uso do suporte ventilatório com pressão positiva contínua em vias aéreas (CPAP) por meio de máscara nasofacial no tratamento da insuficiência respiratória aguda
}

\author{
M.A. Scarpinella-Bueno, C.M. Llarges, A.M. Isola, M.A. Holanda, R.T. Rocha, J .E. Afonso \\ Disciplina de Pneumologia, Universidade Federal de São Paulo - Escola Paulista de Medicina, São Paulo, SP.
}

\begin{abstract}
RESUMO - Овj etıvo. O objetivo dos autores foi avaliar o efeito da ventilação com CPAP oferecida por meio de máscara nasofacial como método de suporte ventilatório em pacientes com insuficiência respiratória aguda com critérios de indicação para intubação traqueal.

Casuística e Método. Foram estudados 11 pacientes com idade média de 41,3 anos em insuficiência respiratória aguda internados na Unidade Respiratória do Hospital São Paulo - Escola Paulista de Medicina. À admissão, era colhida gasometria arterial em ar ambiente e monitorizava-se freqüência respiratória (f), freqüência cardíaca (FC) e pressão arterial (PA). Os mesmos parâmetros eram avaliados após oxigenoterapia via máscara facial aberta e com máscara facial de CPAP usando PEEP de $5 \mathrm{~cm} \mathrm{H}_{2} O$.
\end{abstract}

\section{INTRODUÇÃO}

O interesse pelo suporte ventilatório não-invasivo vem ressurgindo há cerca de 15 anos com o uso de ventiladores com pressão negativa empregados de modo intermitente (particularmente à noite) nos pacientes com sintomas de hipoventilação crôni ca ${ }^{1}$. No início dos anos oitentas, começou-se a empregar pressão positiva nas vias aéreas por mei o de máscaras naso ou or ofaciais em pacientes portadores de insuficiência respiratória aguda e crônica agudizada, tendo-se obtido êxito em evitar a intubação traqueal e em diminuir o número de episódios de agudização de insuficiência respiratória ${ }^{1-5}$.

O uso de pressão positiva contínua em vias aéreas (CPAP), através de máscaras nasofaciais, tem-se mostrado capaz de diminuir tanto o trabaIho inspiratório quanto o expiratório, mel horando a eficiência diafragmática e evitando a estafa muscular ${ }^{6}$.

Se a estafa muscular já estiver instalada, há indicação de intubação traqueal e ventilação mecânica invasiva. Nos pacientes com insuficiência
Resultados. Com o uso de CPAP através de máscara nasofacial, houve melhora significativa dos níveis de $\mathrm{PaO}_{2}$ e diminuição da freqüência respiratória $(p<0,05)$, quando comparados aos valores em ar ambiente e com máscara facial aberta.

Conclusão. Este trabalho permitiu concluir que a máscara facial de CPAP com $5 \mathrm{~cm} \mathrm{H}_{2} \mathrm{O}$ foi eficiente em melhorar a oxigenação arterial e diminuir a freqüência respiratória dos pacientes com insuficiência respiratória aguda, proporcionando-lhes maior conforto, constituindo uma medida terapêutica capaz de evitar o suporte ventilatório invasivo.

UNITERMOS: Máscara facial. CPAP. Insuficiência respiratória aguda.

respiratória aguda (IRpA), a el evação progressiva da pressão arterial de $\mathrm{CO}_{2}\left(\mathrm{PaCO}_{2}\right)$ ainda é, dentre os exames laboratoriais, o mais prático indicativo de insucesso das medidas não-agressivas de suporte ventilatório.

O objetivo deste estudo foi avaliar a eficiência do CPAP aplicado através de máscara nasofacial em paci entes portadores de IRpA, sem doença pulmonar prévia e preenchendo os critérios de indicação de ventilação mecânica invasiva com intubação naso ou or otraqueal. Nas tabelas 1 e 2 , 


\begin{tabular}{|l|}
\hline $\begin{array}{l}\text { Tabela } 2 \text { - Indicações e complicações do uso de CPAP naso } \\
\text { ou orofacial }\end{array}$ \\
$\begin{aligned} \text { Indicações: pacientes com hipoxemia moderada a grave capazes } \\
\text { de ventilar espontaneamente }\end{aligned}$ \\
$\begin{aligned} & \text { Complicações: } \text { hipercapnia } \\
& \text { lesões faciais } \\
& \text { gastrectasia } \\
& \text { vômitos } \text { arritmia cardíaca } \\
& \text { pneumotórax }\end{aligned}$ \\
\hline
\end{tabular}

\begin{tabular}{|c|c|c|c|c|c|}
\hline & Sexo & Idade & Morbidade & $\begin{array}{c}\text { Doenças } \\
\text { associadas }\end{array}$ & $\begin{array}{c}\text { Tempo } \\
\text { de uso } \\
\text { (h) }\end{array}$ \\
\hline 1 & $M$ & 26 & Pneumocistose & AIDS & 48 \\
\hline 2 & $\mathrm{~F}$ & 25 & Pneumonia & & 24 \\
\hline 3 & $\mathrm{~F}$ & 84 & EAP & ICC & 48 \\
\hline 4 & $M$ & 15 & Pneumonia & Estafilococcemia & 24 \\
\hline 5 & $\mathrm{~F}$ & 21 & Pneumonia & Empiema pleural & 18 \\
\hline 6 & $\mathrm{M}$ & 46 & Pneumonia & Tabagismo & 72 \\
\hline 7 & $\mathrm{~F}$ & 27 & Pneumonia & SARA & 48 \\
\hline 8 & $\mathrm{M}$ & 70 & EAP & $\mathrm{ICC}$ & 48 \\
\hline 9 & $\mathrm{M}$ & 43 & Pneumonia & Pancreatite & 72 \\
\hline 10 & M & 22 & $\begin{array}{l}\text { Pneumonia } \\
\text { intersticial }\end{array}$ & Pancreatite & 72 \\
\hline 11 & $\mathrm{~F}$ & 76 & EAP & ICC & 48 \\
\hline
\end{tabular}

apresentamos as principais indicações e complicações do uso de CPAP.

\section{CASUÍSTICA E MÉTOdOS}

O estudo foi realizado na Unidade Respiratória do Hospital São Paulo - Disciplina de PneumoIogi a da E scola Paulista de Medicina - entre abril e dezembro de 1994. F oram estudados onze pacientes (seis homens e cinco mulheres), com idade variando entre 15 e 84 anos (média, 41,3 anos), em insuficiência respiratória aguda, refratários à oxigenoterapia e às medidas gerais. As características dos pacientes quanto ao sexo, idade, diagnóstico e tempo em que o CPAP foi utilizado estão resumidas na tabela 3 .

À admissão eram colhidos, além dos exames pertinentes a cada caso, sangue arterial para determinação de gasometria em ar ambiente e monitorizava-se a freqüência respiratória (f), freqüência cardíaca (FC) e pressão arterial (PA). Em todos os pacientes, foi administrada oxigenoterapia complementar via máscara facial aberta com fluxo de $5 \mathrm{~L} / \mathrm{min}$, além do tratamento específico da moléstia de base. Meia a uma hora após, determinavam-se, novamente, gasometria arterial, f, FC e PA. Apesar da melhora da oxigenação, os pacientes apresentaram evidências de aumento do trabalho respiratório: piora objetiva e subjetiva da dispnéia, retração intercostal e supraesternal, uso de musculatura acessória, piora da freqüência respiratória, sudorese, agitação, tendo sido indicado suporte ventilatório com CPAP nasofacial.

O sistema de CPAP utilizado era composto de máscara facial ajustada ao contorno do rosto e que não permitia vazamento de gás, conectada por uma traquéia a um gerador de fluxo e a uma válvula de PEEP de $5 \mathrm{~cm} \mathrm{H}_{2} \mathrm{O}$ (Vital Signs Inc., Totowa, NJ, EUA). A fração inspirada de $\mathrm{O}_{2}\left(\mathrm{FiO}_{2}\right)$ fornecida por cateter sob a máscara era ajustada a fim de se obter uma saturação de oxigênio maior ou igual a $90 \%$. O gerador de fl uxo utilizado era ligado a uma fonte de ar comprimido com fluxômetro aberto em 15 litros por minuto (L/min), tendo, assim, capacidade de gerar fluxos de 100L/min. Gasometrias arteriais foram realizadas após uma hora de instalada a máscara de CPAP e, posteriormente, pelo menos uma vez ao dia, até a retirada da máscara, que, em nosso estudo, variou de 18 a 72 horas.

Todos os pacientes admitidos no estudo estavam conscientes, alertas e cooperativos, não apresentavam vômitos, broncorréia, íleo adinâmico ou hipercapnia.

Os pacientes serviram como seus próprios controles e a análise estatística foi feita através do teste t de Student para amostras pareadas. Houve significância quando $\mathrm{p}<0,05$.

\section{RESULTADOS}

As tabelas 4 e 5 mostram os resultados de $\mathrm{PaO}_{2}$, $\mathrm{PaCO}_{2}$ e freqüência respiratória dos pacientes em ar ambiente, após oxigenoterapia com a máscara facial convencional e com CPAP com máscara nasofacial usando pressão positiva expiratória de $5 \mathrm{~cm}$ $\mathrm{H}_{2} \mathrm{O}$. O tempo de uso da máscara de CPAP variou entre 18 e 72 horas.

Três pacientes necessitaram de suporte ventilatório mecânico invasivo durante a evolução do estudo. O paciente no 7, após 48 horas de mel hora clínica e gasométrica com o CPAP, voltou a ficar taquipnéico, hipoxêmico e apresentou, ao RX de tórax, infiltrado interstício-alveolar sugestivo de síndrome da angústia respiratória do adulto 
Tabela 4 - Valores de $\mathrm{PaO}_{2}(\mathrm{mmHg}), \mathrm{PaCO}_{2}(\mathrm{mmHg})$ e freqüência respiratória (f) em respirações/minuto em ar ambiente (AA), máscara aberta de $\mathrm{O}_{2}$ e CPAP nasofacial (PEEP $5 \mathrm{~cm} \mathrm{H}_{2} \mathrm{O}$ )

\begin{tabular}{|c|c|c|c|c|c|c|c|c|c|}
\hline & A & A & & áscara a & berta d & $\mathrm{le} \mathrm{O}_{2}$ & CPAP & nasofac & cial \\
\hline & $\mathrm{PaO}_{2}$ & $\mathrm{PaCO}_{2}$ & 2 & $\mathrm{PaO}_{2}$ & $\mathrm{PaCO}_{2}$ & $f$ & $\mathrm{PaO}_{2}$ & $\mathrm{PaCO}_{2}$ & $2 f$ \\
\hline 1 & 35,4 & 30,0 & 70 & 50,6 & 37,0 & 70 & 122,9 & 37,0 & 48 \\
\hline 2 & 52,9 & 23,8 & 52 & 67,6 & 28,5 & 44 & 88,7 & 27,7 & 34 \\
\hline 3 & 83,1 & 28,0 & 40 & 124,6 & 33,0 & 44 & 100,2 & 38,2 & 24 \\
\hline 4 & 52,1 & 29,4 & 48 & 53,1 & 41,0 & 48 & 122,0 & 33,0 & 36 \\
\hline 5 & 62,6 & 25,4 & 40 & 95,8 & 30,6 & 38 & 105,0 & 30,9 & 24 \\
\hline 6 & 56,9 & 33,7 & 24 & 42,3 & 27,8 & 36 & 92,3 & 33,2 & 26 \\
\hline 7 & 54,8 & 31,7 & 32 & 59,8 & 32,8 & 48 & 197,2 & 26,4 & 32 \\
\hline 8 & 59,4 & 20,5 & 52 & 79,9 & 22,5 & 52 & 103,0 & 21,1 & 24 \\
\hline 9 & 56,4 & 25,1 & 44 & 70,7 & 24,0 & 44 & 156,2 & 29,2 & 24 \\
\hline 10 & 46,7 & 24,1 & 58 & - & - & - & 96,9 & 38,9 & 48 \\
\hline 11 & 58,0 & 25,2 & 24 & 59,5 & 35,9 & 24 & 92,2 & 35,0 & 22 \\
\hline 入 & 56,2 & 27,0 & 43,3 & 70,2 & 30,8 & 45,6 & 116,0 & 31,8 & 30,9 \\
\hline d & $\pm 11,0$ & $\pm 3,7 \pm$ & $\pm 13,4$ & $\pm 22,1$ & $\pm 5,5$ & $\pm 11,0$ & $\pm 31,7$ & $\pm 5,1$ & $\pm 9,1$ \\
\hline
\end{tabular}

Tabela 5 - Análise estatística entre os valores de $\mathrm{PaO}_{2}$ $(\mathrm{mmHg}), \mathrm{PaCO}_{2}(\mathrm{mmHg})$ e freqüência respiratória $(\mathrm{f}) \mathrm{em}$ respirações por minuto obtidos em ar ambiente (AA), máscara aberta de $\mathrm{O}_{2}$ e CPAP nasofacial

$$
\begin{array}{lll}
\mathrm{PaO}_{2} & -\mathrm{AA} \times \text { máscara de } \mathrm{O}_{2}-\mathrm{p}=0,007^{*} \\
\mathrm{PaCO}_{2} & -\mathrm{AA} \times \text { máscara de } \mathrm{O}_{2}-\mathrm{p}=0,01^{*} \\
\mathrm{f} & -\mathrm{AA} \times \text { máscara de } \mathrm{O}_{2}-\mathrm{p}=0,1 \\
\mathrm{PaO}_{2} & -\mathrm{AA} \times \mathrm{CPAP}-\mathrm{p}=0,0001^{*} \\
\mathrm{PaCO}_{2} & -\mathrm{AA} \times \mathrm{CPAP}-\mathrm{p}=0,007^{*} \\
\mathrm{f} & -\mathrm{AA} \times \mathrm{CPAP}-\mathrm{p}=0,0001^{*} \\
\mathrm{PaO}_{2} & - \text { máscara de } \mathrm{O}_{2} \times \mathrm{CPAP}-\mathrm{p}=0,002^{*} \\
\mathrm{PaCO}_{2} & - \text { máscara de } \mathrm{O}_{2} \times \mathrm{CPAP}-\mathrm{p}=0,2 \\
\mathrm{f} & - \text { máscara de } \mathrm{O}_{2} \times \mathrm{CPAP}-\mathrm{p}=0,00003^{*}
\end{array}
$$

* Significativo quando $p<0,05$

(SARA) $)^{7}$. O paciente no 8,24 horas após ter iniciado o uso do CPAP, apresentou hemoptise moderada. Como persistia desconfortável do ponto de vista respiratório, apesar da melhora da $\mathrm{PaO}_{2}$ e diminuição na freqüência respiratória, optou-se por ventilação mecânica. À broncoscopia, observou-se sangramento difuso da mucosa de segmento anterior de lobo superior esquerdo. A progressão para quadro de SARA também foi observada no paciente no 10 , sendo indicada intubação traqueal após 48 horas de iniciado o estudo. Desses três pacientes, os dois que evoluíram com SARA apresentaram sepse e posterior insuficiência de múltiplos órgãos e sistemas, vindo a falecer.
Houve melhora significativa na $\mathrm{PaO}_{2}$, como era esperado, ao se comparar os valores em ar ambiente com o CPAP nasofacial $(56,2 \pm 11 \mathrm{mmH}$ g e 116 $\pm 31,7 \mathrm{mmHg}$, respectivamente). Quando comparamos os níveis arteriais de oxigênio nos pacientes com máscara aberta de $\mathrm{O}_{2}$ e CPAP através de máscara nasofacial, observamos que esses últimos apresentaram melhora significativa (70,2 \pm $22,1 \mathrm{mmHg}$ e $116 \pm 31,7 \mathrm{mmHg}$, respectivamente) $(p<0,05)$.

O uso da máscara de CPAP relacionou-se não só ao menor uso da musculatura acessória e diminuição de retrações intercostais e supra-esternal, mas, também, a uma diminuição significativa da freqüência respiratória, quando comparada com a máscara convencional de $\mathrm{O}_{2}$ ou em ar ambiente $(43,3 \pm 13,4 \mathrm{i} / \mathrm{min}$ em ar ambiente e 45,6 $\pm 11 \mathrm{i} / \mathrm{min}$ com máscara aberta e 30,9 $\pm 9,1 \mathrm{i} / \mathrm{min}$ com CPAP) $(p<0,05)$.

Não houve retenção significativa de $\mathrm{CO}_{2}$ nos pacientes que utilizaram a máscara de CPAP $(p<0,05)$. Alterações significativas de PA e freqüência cardíaca não foram observadas.

$\mathrm{N}$ os pacientes que se beneficiaram com o uso da pressão positiva em vias aéreas, as gasometrias arteriais colhidas entre uma e uma hora e trinta minutos após a retirada da máscara de CPAP mostraram níveis de $\mathrm{PaO}_{2}$ esperados para a idade, ausência de hipercarbia e freqüência respiratória dentro dos limites normais.

Todos os pacientes submetidos ao estudo toleraram bem o procedimento. Não foram notados distensão gástrica, pneumotórax ou necrose cutânea que pudessem interromper o uso da máscara de CPAP.

\section{DISCUSSÃO}

A máscara facial com pressão positiva contínua em vias aéreas tem sido usada em pacientes com insuficiência respiratória aguda, tanto de origem cardíaca quanto pulmonar, em particular nos casos de hipoxemia com aumento do trabalho respiratório ${ }^{1-3}$. Tais pacientes apresentam parâmetros próximos ou dentro dos critérios de indicação de suporte ventilatório convencional, porém, obedecendo aos critérios de inclusão para o uso da máscara de CPAP (tabela 1$)^{8}$, beneficiar-se-ão em muito deste modo de ventilação, seja pelo maior conforto, pela menor necessidade de sedação, pela manutenção das defesas de vias aéreas, seja para se evitar as complicações de uma intubação traqueal ${ }^{1,8,9}$. Essas complicações podem estar relacionadas ao procedimento em si (traumatismo durante a passagem do tubo, hemorragias, má loca- 
lização do tubo, lesão glótica), ao uso da pressão positiva (barotrauma, diminuição do retorno venoso), ao uso de sedativos e relaxantes musculares e alterações em diversos órgãos e sistemas, tais como os rins (hipoperfusão), o aparel ho digestivo (distensão gástrica e íleo paralítico) e o sistema nervoso central (hipertensão intracraniana) ${ }^{10,11}$.

Rasanen et al. ${ }^{2}$ estudaram a eficiência terapêutica da máscara facial de CPAP em 40 pacientes com edema agudo pulmonar cardiogênico e IRpA. Inicialmente, os pacientes receberam suplementação de oxigênio através de máscara aberta e, posteriormente, foram, aleatoriamente, separados em dois grupos, a saber, 20 paci entes receberam $10 \mathrm{~cm}$ $\mathrm{H}_{2} \mathrm{O}$ de CPAP e 20 continuaram a receber $\mathrm{O}_{2}$ sob pressão ambiente. O uso do CPAP melhorou significativamente a troca gasosa, diminuiu o trabalho respi ratório e reduzi u a necessidade de ventilação invasiva para o tratamento do edema pulmonar cardiogênico. Outros estudos com resultados semel hantes são relatados na literatura ${ }^{1,12}$.

E m pacientes com AIDS e pneumocistose, a máscara facial de CPAP tem-se mostrado alternativa eficaz em melhorar a oxigenação quando há hipoxemia severa, em particular naqueles casos em que a pneumonia por $\mathrm{P}$. carini i já ocorreu anteriormente, sendo discutida a indicação de intubação traqueal ${ }^{8,9}$.

A hipoxemia apresentada por nossos pacientes pode ser explicada, fundamentalmente, por três mecanismos, a saber, shunt, desigualdade da relação ventilação-perfusão ( $V / Q)$ e diminuição da difusão de oxigênio. Essas alterações fisiopatológi cas são observadas nas lesões inflamatórias, pneumônicas, SARA e edema pulmonar de qualquer eti ologia, até mesmo cardi ogênico. São, também, provocadas e/ou agravadas por fatores que aumentam a resistência das vias aéreas e diminuem a complacência $a^{2,3,9,12}$.

O uso de CPAP nasofacial com máscara melhora a pressão de fluxo inspiratório devido à elevação da pressão de nasofaringe, e O PEEP aumenta a capacidade residual funcional por meio da reexpansão de alvéolos colapsados e hipoinsuflados, melhorando a ventilação em áreas de baixo V/Q. Isto leva à diminuição da hipoxemia por redução do shunt e do efeito shunt. O aumento da capacidade residual funcional eleva a complacência e, portanto, diminui o trabalho respiratório ${ }^{1-3,5,12}$.

Provavelmente, a manutenção de trocas gasosas adequadas em nossos paci entes após a retirada do CPAP deveu-se à melhora das alterações pulmonares e à dimi nui ção do trabal ho respi ratório, que, em nosso estudo, pôde ser subjetivamente avaliada pela diminuição da freqüência respiratória e do uso da musculatura acessória. Em pacientes portadores de doença pulmonar obstrutiva crônica (DPOC), essa diminuição do trabalho respiratório com o uso do CPAP está bem estabelecida e parece ser decorrente do seu efeito sobre os músculos inspiratórios, melhorando a hiperinsuflação dinâmica e a compressão das vias aéreas ${ }^{13,14}$.

O CPAP nasofacial também tem sido usado com êxito nos pacientes com IRp crônica agudizada; acredita-se que, nesses casos, a hipoxemia seja corrigida mediante um destes três mecanismos: alívio da fadiga muscular, melhora da complacência torácica por meio da reversão de microatelectasias e impedimento da chamada fadiga central, um processo de adaptação por parte do centro respiratório a fim de manter um trabal ho respiratório acima do limiar que precipitaria à fadiga muscular ${ }^{1,4}$.

As principais limitações do CPAP incluem a necessidade da cooperação pelo paciente, até mesmo coordenando seus esforços respiratórios com a geração de altos fluxos, ocorrência de atelectasias por "rolhas" de secreção, úlceras faciais, aerofagia e vômi tos ${ }^{1,3}$. Nenhum de nossos pacientes apresentou tais complicações.

Meyer e $\mathrm{Hill}^{1}$ definem como taxa de sucesso para ventilação não-invasiva com pressão positiva a percentagem de pacientes que toleram o procedimento, tendo melhora na hematose e evitando a necessi dade de intubação traqueal. E m nosso estudo, a taxa de sucesso foi de $72,7 \%$. Se considerarmos que dos 11 pacientes, um del es era portador de AIDS e três tinham idade superior a 70 anos, observamos que a máscara de CPAP se torna um método terapêutico eficiente em melhorar a oxigenação em pacientes com hipoxemia severa.

Meduri et al ${ }^{15}$, em estudo com ventilação com máscara facial em pacientes com IRp crônica agudizada, observaram que os melhores preditores de insucesso na técnica recaem na falta de melhora das trocas gasosas ou, então, na persistência da taquipnéia após duas horas de iniciado o procedimento.

Apesar de não termos utilizado grupo controle em nosso estudo, se não administrássemos o CPAP por meio de máscara nasofacial, tais pacientes teriam sido submetidos à intubação traqueal e ventilação convencional. Concluímos que O CPAP nasofacial com $5 \mathrm{~cm} \mathrm{H_{2 }}$ O de PEEP foi eficiente em melhorar a oxigenação arterial com $\mathrm{FiO}_{2}$ mais baixas e diminuir a freqüência respiratória de pacientes Com IRpA, diminuindo a necessidade de suporte ventilatório invasivo, constituindo-se em uma "ponte" segura entre a máscara facial aberta de $\mathrm{O}_{2}$ e o ventilador mecânico. 
A instabilidade clínica própria dos pacientes em IRpA e o fato de a resposta terapêutica da doença de base ser imprevisível, aliados à possibilidade de má tolerância e até resposta inadequada ao CPAP, tornam obrigatório o monitoramento clínico e laboratorial durante a sua utilização.

\section{SUMMARY}

\section{Continuous positive airway pressure (CPAP) by face mask to treat acute respiratory failure}

OBjeCtIVE. To evaluate the efficacy of continuous positive airway pressure (CPAP) administered by face mask in patients with acute respiratory failure with indication of mechanical ventilation.

Materials and Method. Eleven patients (mean age 41.3 years) in acute respiratory failure were admitted in the Respiratory Unit - Hospital São Paulo - Escola Paulista de Medicina. At the admission $\mathrm{pH}, \mathrm{PaO}_{2}, \mathrm{PaCO}_{2}$, respiratory rate (f), arterial pressure and heart rate were measured. The measurements were repeated with administration of oxigen with a high flow face mask at ambient airway pressure and then with $5 \mathrm{~cm} \mathrm{H}_{2} \mathrm{O}$ of CPAP by face mask.

RESULTS. The use of CPAP by face mask significantly improved the arterial blood oxigenation and decreased the respiratory rate $(p<0.05)$.

Conclusion. These data allow the conclusion that CPAP administered by face mask improves gas exchange and decreases respiratory rate in patients with acute respiratory failure and may re duce the need for invasive mechanical ventilation. [Rev Ass Med Brasil 1997; 43(3): 180-4]

KEY WORDS: Face mask. CPAP. Acute respiratory failure.

\section{REFERÊNCIAS BIBLIOGRÁFICAS}

1. Meyer TJ , Hill NS. Noninvasive positive pressure ventilation totreat respiratory failure. Ann Intern Med 1994; 120: 760-70.

2. Rasanen J, Heikkila J, Downs J, Nikki P, Vaisanen I, Viitanen A. Continuous positive airway pressure by face mask in acute cardiogenic pulmonary edema. Am J Cardiol 1985; 55: 296-300.

3. Brett A, Sinclair DG. Use of continuous positive airway pressure in the management of community acquired pneumonia. Thorax 1993; 48: 1.280-1.

4. Benhamou D, Girault C, F aureC, Portier F, Muir J F. Nasal mask ventilation in acute respiratory failure. Chest 1992; 102: 912-7.

5. Lucas $\mathrm{P}$, Tarácon $\mathrm{C}$, Puente $\mathrm{L}$ et al. Nasal continuous positive airway pressure in patients with COPD in acute respiratory failure. Chest 1993; 104: 1.694-7.

6. O'Donnell, Sani R, Younes M. Improvement in exercise endurance in patients with chronic airflow limitation using continuous positiveairway pressure. Am Rev Respir Dis 1988; 138: 1510-4.

7. Murray J F, Matthay MA, Luce J M, Flick MR. An expanded definition of the adult respiratory distress syndrome. Am Rev Respir Dis 1988; 138: 720-3.

8. DeVita MA, Friedman Y, Petrella V. Pressão respiratória positiva contínua por máscara nos pacientes aidéticos. Clin Med Am Norte 1993; 4: 135-49.

9. Miller RF, SempleSJ G. Continuous positiveairway pressure ventilation for respiratory failure associated with Pneumocystis carinii pneumonia. Resp Med 1991; 85: 133-8.

10. Parker J C, Hernandez LA, Peevy KJ. Mechanisms of ventilator-induced lung injury. Crit Care Med 1993; 21: 131-43.

11. Slutsky AS. Mechanical ventilation. Chest 1993; 104: 1.833-59.

12. Vaisanen IT, Rasanen J . Continuous positiveairway pressure and supplemental oxigen in the treatment of cardiogenic pulmonary edema. Chest 1987; 92: 481-5.

13. O'Donnell DE, Sanii R, Giesbrecht G, Younes, M. Effect of continuous positive airway pressure on respiratory sensation in patients with chronic obstructive pulmonary diseaseduring submaximal exercise. Am Rev Respir Dis 1988; 138: 1.185-91.

14. Smith TC, Marini J J . I mpact of PEEP on lung mechanics and work of breathing in severe airflow obstruction. J. Appl. Physiol 1988; 65: 1.488-99.

15. Meduri GU, Conoscenci CC, Menashe P, Nair S. Noninvasive face mask ventilation in patients with acute respiratory failure. Chest 1989; 95: 865-70. 